\title{
Bulk Rashba Effect Splitting and Suppression in Polymorphs of Metal lodine Perovskites
}

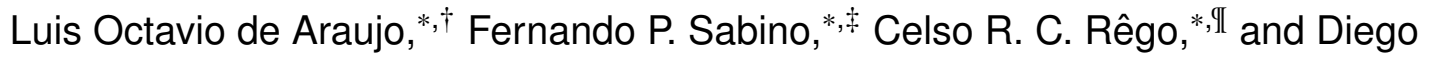

\author{
Guedes-Sobrinho*,†
}

$†$ Chemistry Department, Federal University of Paraná, CEP 81531-980, Curitiba, Brazil

$\ddagger$ Federal University of ABC, Santo André, São Paulo, 09210-580, Brazil

IInstitute of Nanotechnology Hermann-von-Helmholtz-Platz, Karlsruhe Institute of Technology, 76021 Karlsruhe, Germany

E-mail: luisaraujo@ufpr.br; fernandopsabino@yahoo.com.br; celso.rego@kit.edu; guedessobrinho@ufpr.br 


\section{DFT-1/2 Quasiparticle Correction Method}

The gap energies of all systems were calculated through the quasiparticle correction named DFT-1/2, which has been implemented through codes as WIEN2K, ${ }^{1}$ VASP,${ }^{2}$ and ExcITING, ${ }^{3}$ as well as has been applied to the prediction (with excellent precision) of a large number of materials. ${ }^{2,4-7}$ DFT-1/2 method stems from Slater transition technique ${ }^{8}$ from which the gap energy inaccuracy is reduced through the half electron/half hole occupation, whose atomic eigenvalue is equal to the atomic ionization potential. Ferreira et al. ${ }^{1}$ extended to DFT for crystalline systems, from which the half-occupation is associated to a modified Kohn-Sham potential as $V_{\text {mod,KS }}=V_{\mathrm{KS}}(\vec{r})-V_{S}(\vec{r})$, where $V_{\mathrm{KS}}(\vec{r})$ is the standard Kohn-Sham potential and $V_{S}(\vec{r})$ is the self-energy potential. The $V_{S}(\vec{r})$ is given by the difference between the Kohn-Sham potentials for the neutral (all-electron, ae) and half-ionized (ae-1/2) atoms. To avoid the interpenetration of the self-energy Coulomb tails into neighboring atom sites, the self-energy potential are trimmed by a function $\Theta(\vec{r}, C U T)$, that is

$$
V_{S}(\vec{r})=\Theta(\vec{r}, C U T)\left[V_{a e}(\vec{r})-V_{a e-1 / 2}(\vec{r})\right] .
$$

from which the $C U T$ parameter is determined variationally through the maximization of the gap energy without taking into account empirical parameters.

We performed bandgap energy $\left(E_{g}\right)$ calculations for all pure perovskites in cubic, tetragonal, and orthorhombic structures through the DFT-1/2 approach. The character of the top of the valence band was analyzed to define the potential to be corrected. As such, $\mathrm{MAPbI}_{3}, \mathrm{MASnI}_{3}, \mathrm{MAGeI}_{3}$, and $\mathrm{MASiI}_{3}$ perovskites are composed by $s-p$ states which have the $5 p$ state from I as majority contribution into $\mathrm{PbI}_{3}{ }^{-}, \mathrm{SnI}_{3}{ }^{-}, \mathrm{GeI}_{3}{ }^{-}$, and $\mathrm{SiI}_{3}{ }^{-}$inorganic lattices. Table $\mathrm{S} 1$ summarizes all $E_{g}$ calculated by means DFT, DFT+SOC, and DFT-1/2 without SOC by presenting their respective CUT $\left(a_{0}\right)$ values. Experimental data are indicated for perovskites into crystalline phase already synthesized. Our results show an invariance of the $C U T$ parameter with respect to the structure of a particular chemical composition, e.g., for $\mathrm{MAPbI}_{3}$, in which the energy gap maximization lies on $C U T=3.88 a_{0}$ for cubic, tetragonal, and orthorhombic structures. $\mathrm{MASnI}_{3}, \mathrm{MAGeI}_{3}$, and $\mathrm{MASiI}_{3}$ present the same behaviour for their respective $C U T$ parameters for all structures.

\section{Structure Properties}

We analyzed atomic size effects for $\mathrm{Sn}, \mathrm{Ge}$, and $\mathrm{Si}$ with respect to the $\mathrm{Pb}$ size, which are smaller in 4.08, 17.01, and $32.65 \%$, respectively. ${ }^{19}$ The sequence of metal size in $\mathrm{Pb}>\mathrm{Sn}>\mathrm{Ge}>\mathrm{Si}$, i.e., diminishing the atomic number in the IV group of the periodic table, correlates with the decreasing of lattice parameters and unit cell volume. As such, with respect to the $\mathrm{MAPbI}_{3}$, the maximum contraction of the lattice parameters is for $\mathrm{MASiI}_{3}$, which after stress tensor relaxation its unit cell volume reduces by $14.29 \%, 8.71 \%$, and $17.66 \%$ for pseudo-cubic, tetragonal, and orthorhombic 
Table S1 - Gap energies (in eV) from calculation protocols from DFT standard (without SOC), DFT+SOC, and DFT-1/2 (without SOC) in comparison with experiments for MAPbI ${ }_{3}, \mathrm{MASnI}_{3}, \mathrm{MAGeI}_{3}$, and $\mathrm{MASiI}_{3}$ perovskites in cubic $(\alpha)$, tetragonal $(\beta)$, and orthorhombic $(\gamma)$.

\begin{tabular}{lcccccc}
\hline & DFT & DFT+SOC & DFT-1/2 & DFT-1/2+SOC & exp. & CUT $\left(a_{0}\right)$ \\
\hline$\alpha-\mathrm{MAPbI}_{3}$ & 1.65 & 0.61 & 2.64 & 1.50 & $1.48^{9}$ & 3.88 \\
$\alpha-\mathrm{MASnI}_{3}$ & 0.70 & 0.43 & 1.60 & 1.26 & $1.30^{10,11}$ & 3.70 \\
$\alpha-\mathrm{MAGeI}_{3}$ & 1.22 & 1.10 & 2.02 & 1.90 & $1.90^{12}$ & 3.70 \\
$\alpha-\mathrm{MASiI}_{3}$ & 0.87 & 0.81 & 1.50 & 1.44 & & 3.46 \\
\hline & & & & & & \\
\hline$\beta-\mathrm{MAPbI}_{3}$ & 1.60 & 0.71 & 2.61 & 1.66 & $1.62^{13-16}$ & 3.88 \\
$\beta-\mathrm{MASnI}_{3}$ & 0.68 & 0.47 & 1.56 & 1.32 & $1.21^{17}$ & 3.70 \\
$\beta-\mathrm{MAGeI}_{3}$ & 1.26 & 1.15 & 2.04 & 1.94 & & 3.70 \\
$\beta-\mathrm{MASiI}_{3}$ & 0.91 & 0.85 & 1.53 & 1.48 & & 3.46 \\
\hline & & & & & & \\
\hline$\gamma-\mathrm{MAPbI}_{3}$ & 1.76 & 0.72 & 2.75 & 1.68 & 1.68 & 3.88 \\
$\gamma-\mathrm{MASnI}_{3}$ & 0.80 & 0.48 & 1.67 & 1.33 & & 3.70 \\
$\gamma-\mathrm{MAGeI}_{3}$ & 0.86 & 0.64 & 1.65 & 1.44 & & 3.70 \\
$\gamma-\mathrm{MASiI}_{3}$ & 0.09 & 0.02 & 0.78 & 0.69 & & 3.46 \\
\hline
\end{tabular}

Table S2 - Spin-orbit coupling energies for the metals and iodine in the MAPbI ${ }_{3}, \mathrm{MASnI}_{3}, \mathrm{MAGeI}_{3}$, and $\mathrm{MASiI}_{3}$ perovskites in cubic $(\alpha)$, tetragonal $(\beta)$, and orthorhombic $(\gamma)$.

\begin{tabular}{lcc}
\hline & \multicolumn{2}{c}{$E_{\mathrm{SOC}}(\mathrm{meV})$} \\
\hline & Metal & Iodine \\
\hline$\alpha-\mathrm{MAPbI}_{3}$ & -972 & -330 \\
$\alpha-\mathrm{MASnI}_{3}$ & -142 & -340 \\
$\alpha-\mathrm{MAGeI}_{3}$ & -24 & -340 \\
$\alpha-\mathrm{MASiI}_{3}$ & 0 & -340 \\
\hline & & \\
\hline$\beta-\mathrm{MAPbI}_{3}$ & -1180 & -340 \\
$\beta-\mathrm{MASnI}_{3}$ & -140 & -340 \\
$\beta-\mathrm{MAGeI}_{3}$ & -24 & -340 \\
$\beta-\mathrm{MASiI}_{3}$ & 0 & -340 \\
\hline \multicolumn{3}{l}{} \\
\hline$\gamma-\mathrm{MAPbI}_{3}$ & -1170 & -340 \\
$\gamma-\mathrm{MASnI}_{3}$ & -138 & -330 \\
$\gamma-\mathrm{MAGeI}_{3}$ & -24 & -330 \\
$\gamma-\mathrm{MASiI}_{3}$ & 0 & -340 \\
\hline
\end{tabular}

structures, respectively.

For the tetragonal structure in a given layer, the octahedrons tilt (M-I-M angle in Table S3) in an opposite direction with respect to the neighbor layer, while in orthorhombic the tilting between them is in matching - compare the representations for tetragonal and orthorhombic structures from the $a c$ and $a b$ planes point of view. As consequence, a larger contraction (17.66\%) going from $\mathrm{MAPbI}_{3}$ to $\mathrm{MASiI}_{3}$ is permitted for orthorhombic, while for tetragonal the cavity volume in which $\mathrm{MA}^{+}$cation is accommodated into tetragonal needs to be compensated, yielding only $8.71 \%$ in the unit cell volume. 
Table S3 - Comparative M-I-M angles (deg) in cubic, tetragonal, and orthorhombic structures for the MASiI ${ }_{3}$, $\mathrm{MAGeI}_{3}, \mathrm{MASnI}_{3}$, and MAPbI ${ }_{3}$ perovskites for the fully optimized structures, platonic centrosymmetric (with MA cations relaxed), and platonic tilted centrosymmetric (with MA cations relaxed).

\begin{tabular}{|c|c|c|c|c|c|c|c|c|c|}
\hline \multirow[t]{3}{*}{ System } & \multicolumn{9}{|c|}{ M-I-M angle (deg) } \\
\hline & \multicolumn{3}{|c|}{ Fully relaxed } & \multicolumn{3}{|c|}{ Platonic centrosymmetric } & \multicolumn{3}{|c|}{ Platonic tilted centrosymmetric } \\
\hline & equat. & api. & Tilting & equat. & api. & Tilting & ocunt & api. & Tilting \\
\hline$\alpha-\mathrm{MAPbI}_{3}$ & $167-173$ & $167-167$ & $a^{+} a^{+} a^{+}$ & $180-180$ & $180-180$ & $a^{0} a^{0} a^{0}$ & $161-161$ & $180-180$ & $a^{0} a^{0} a^{+}$ \\
\hline$\alpha-\mathrm{MASnI}_{3}^{3}$ & $169-173$ & $170-170$ & $a^{+} a^{+} a^{+}$ & $180-180$ & $180-180$ & $a^{0} a^{0} a^{0}$ & $168-168$ & $167-180$ & $a^{0} a^{+} a^{+}$ \\
\hline$\alpha-\mathrm{MAGeI}_{3}$ & $166-167$ & $163-163$ & $a^{+} a^{+} a^{+}$ & $180-180$ & $180-180$ & $a^{0} a^{0} a^{0}$ & $154-154$ & $154-180$ & $a^{0} a^{+} a^{+}$ \\
\hline$\alpha-\mathrm{MASiI}_{3}$ & $165-168$ & $164-164$ & $a^{+} a^{+} a^{+}$ & $180-180$ & $180-180$ & $a^{0} a^{0} a^{0}$ & $157-157$ & $157-180$ & $a^{0} a^{+} a^{+}$ \\
\hline$\beta-\mathrm{MAPbI}_{3}$ & $152-157$ & $172-175$ & $a^{0} a^{+} c^{-}$ & $180-180$ & $180-180$ & $a^{0} a^{0} c^{0}$ & $155-155$ & $180-180$ & $a^{0} a^{0} c^{-}$ \\
\hline$\beta$-MASnI 3 & $153-163$ & $171-176$ & $a^{0} a^{+} c^{-}$ & $180-180$ & $180-180$ & $a^{0} a^{0} c^{0}$ & $155-155$ & $180-180$ & $a^{0} a^{0} c^{-}$ \\
\hline$\beta-\mathrm{MAGeI}_{3}^{3}$ & $153-171$ & $165-172$ & $a^{0} a^{+} c^{-}$ & $180-180$ & $180-180$ & $a^{0} a^{0} c^{0}$ & $141-141$ & $180-180$ & $a^{0} a^{0} c^{-}$ \\
\hline$\beta$-MASiI ${ }_{3}$ & $157-173$ & $165-170$ & $a^{0} a^{+} c^{-}$ & $180-180$ & $180-180$ & $a^{0} a^{0} c^{0}$ & $141-141$ & $180-180$ & $a^{0} a^{0} c^{-}$ \\
\hline$\gamma-\mathrm{MAPbI}_{3}$ & $152-152$ & $167-167$ & $a^{-} b^{+} c^{-}$ & $180-180$ & $180-180$ & $a^{0} b^{0} c^{0}$ & $152-152$ & $167-167$ & $a^{-} b^{+} c^{-}$ \\
\hline$\gamma-\mathrm{MASnI}_{3}^{3}$ & $157-157$ & $168-168$ & $a^{-} b^{+} c^{-}$ & $180-180$ & $180-180$ & $a^{0} b^{0} c^{0}$ & $157-157$ & $168-168$ & $a^{-} b^{+} c^{-}$ \\
\hline$\gamma-\mathrm{MAGeI}_{3}$ & $164-164$ & $171-171$ & $a^{-} b^{+} c^{-}$ & $180-180$ & $180-180$ & $a^{0} b^{0} c^{0}$ & $164-164$ & $171-171$ & $a^{-} b^{+} c^{-}$ \\
\hline$\gamma-\mathrm{MASiI}_{3}{ }^{3}$ & 169-169 & $172-172$ & $a^{-} b^{+} c^{-}$ & $180-180$ & $180-180$ & $a^{0} b^{0} c^{0}$ & 169-169 & $172-172$ & $a^{-} b^{+} c^{-}$ \\
\hline
\end{tabular}

\section{Formation Enthalpy}

Formation enthalpies for the polymorphs in $\mathrm{A} \longrightarrow \mathrm{B} \longrightarrow \mathrm{C}$ configurations were calculated from the $i$-th precursors $\mathrm{CH}_{3} \mathrm{NH}_{2(\mathrm{~g})}, \mathrm{HI}_{(\mathrm{g})}$ gas-phase, and $\mathrm{MI}_{2(\mathrm{~s})}$ 2D-layer $(\mathrm{M}=\mathrm{Si}, \mathrm{Ge}, \mathrm{Sn}$, and $\mathrm{Pb})$ as trigonal-phase (3m point group) through $\Delta H_{\mathrm{f}}=E_{\mathrm{MAMI}_{3}}-\sum E_{i}$ approach, i.e., from the $\mathrm{CH}_{3} \mathrm{NH}_{2(\mathrm{~g})}+\mathrm{HI}_{(\mathrm{g})}+\mathrm{MI}_{2(\mathrm{~s})} \rightarrow \mathrm{MAMI}_{3(\mathrm{~s})}$ formation process. For $\Delta H_{\mathrm{f}}$ obtained through the total energies calculations, we considered only the DFT+SOC approach. Numerical values for the $\Delta H_{\mathrm{f}}$ are summarized in Table S4. For the orthorhombic $(\gamma)$ perovskites is important to highlight that its unit cell is metal on-centering, i.e., A polymorph is equal C. $\Delta H_{\mathrm{f}}$ values for $\gamma-\mathrm{MAMI}_{3}$ are also graphically represented in Figure S3 (b), (d), (f), and (h).

\section{Partial Charge Population: DDEC6 vs. Bader Charge Method}

Our results from DFT-1/2+SOC were able to capture the octahedral tilting effects on the bandgap energy opening, as well as the suppression of bulk Rashba from the metal on-centering configurations. Proposing to evaluate polar distortions and their role on the bulk Rashba splitting/suppression at the presence of SOC effects, we applied the density-derived electrostatic and chemical (DDEC6) ${ }^{20}$ and Bader charge ${ }^{21}$ methods for the partial charges mapping of the perovskites in all compositions, structures, and polymorphs. Our DDEC6 results provide a set of charges $\left(\Delta Q_{\mathrm{D}}\right.$ in Figure $\left.1 \mathrm{~S}\right)$ chemically intuitive with reliable atomic charges for this material in consistency with the Pauling electronegativities, ${ }^{22}$ whereas the partial charges calculated via Bader's method ( $\Delta Q_{\mathrm{B}}$ Figure $\left.2 \mathrm{~S}\right)$ wrongly provides positive partial charge 
Table S4 - Numerical values for the $\Delta H_{\mathrm{f}}$ for the MAPbI ${ }_{3}, \mathrm{MASnI}_{3}, \mathrm{MAGeI}_{3}$, and MASiI ${ }_{3}$ perovskites in cubic $(\alpha)$, tetragonal $(\beta)$, and orthorhombic $(\gamma)$ from the $\mathrm{CH}_{3} \mathbf{N H}_{2(\mathrm{~g})}+\mathrm{HII}_{(\mathrm{g})}+\mathrm{MI}_{2(\mathrm{~s})} \rightarrow \mathrm{MAMI}_{3(\mathrm{~s})}$ route, where $\mathrm{M}=\mathrm{Pb}, \mathrm{sn}, \mathrm{Ge}$, and $\mathrm{Si}$.

\begin{tabular}{|c|c|c|c|}
\hline & \multicolumn{3}{|c|}{$\Delta H_{\mathrm{f}}(\mathrm{meV} /$ atom $)$} \\
\hline & A & $\mathrm{B}$ & $\mathrm{C}$ \\
\hline$\alpha-\mathrm{MAPbI}_{3}$ & -150.80 & -146.46 & -150.49 \\
\hline$\alpha-\mathrm{MASnI}_{3}$ & -42.62 & -38.16 & -36.86 \\
\hline$\alpha-\mathrm{MAGeI}_{3}$ & -41.55 & -20.54 & -5.54 \\
\hline$\alpha$-MASiI ${ }_{3}$ & -57.73 & 0.00 & -3.4 \\
\hline$\beta-\mathrm{MAPbI}_{3}$ & -159.10 & -145.81 & -153.71 \\
\hline$\beta$-MASnI ${ }_{3}$ & -48.60 & -39.76 & -44.61 \\
\hline$\beta-\mathrm{MAGeI}_{3}$ & -44.28 & -24.55 & 8.56 \\
\hline$\beta-\mathrm{MASiI}_{3}$ & -59.47 & -21.65 & 20.51 \\
\hline$\gamma-\mathrm{MAPbI}_{3}$ & -158.77 & -141.42 & - \\
\hline$\gamma$-MASnI 3 & -47.62 & -32.70 & - \\
\hline$\gamma-\mathrm{MAGeI}_{3}$ & -31.93 & -22.03 & - \\
\hline$\gamma$-MASiI ${ }_{3}$ & -34.80 & -12.20 & - \\
\hline
\end{tabular}

for carbon atom into $\mathrm{CH}_{3} \mathrm{NH}_{3}{ }^{+}$molecules. We found through DDEC6 a $\Delta Q_{\mathrm{D}}$ cationicity diminishment for the metal with $\mathrm{Pb}>\mathrm{Sn}>\mathrm{Ge}>\mathrm{Si}$, which results in an adjustment of the polarity with the iodine partial charges given the $\mathrm{CH}_{3} \mathrm{NH}_{3}{ }^{+}$dipole moment that keeps constant, i.e., the $\mathrm{CH}_{3}$ and $\mathrm{NH}_{3}$ partial charges are invariant with respect to metal into octahedral sites. The $\mathrm{M}-\mathrm{I}$ the charge polarization are invariant for $\mathrm{A}, \mathrm{B}$, and $\mathrm{C}$ polymorphs in all perovskites and their structures, which confirms the role of the metal off-centering for the bulk Rashba splitting whereas its suppression is reached through the metal on-centering configurations. 


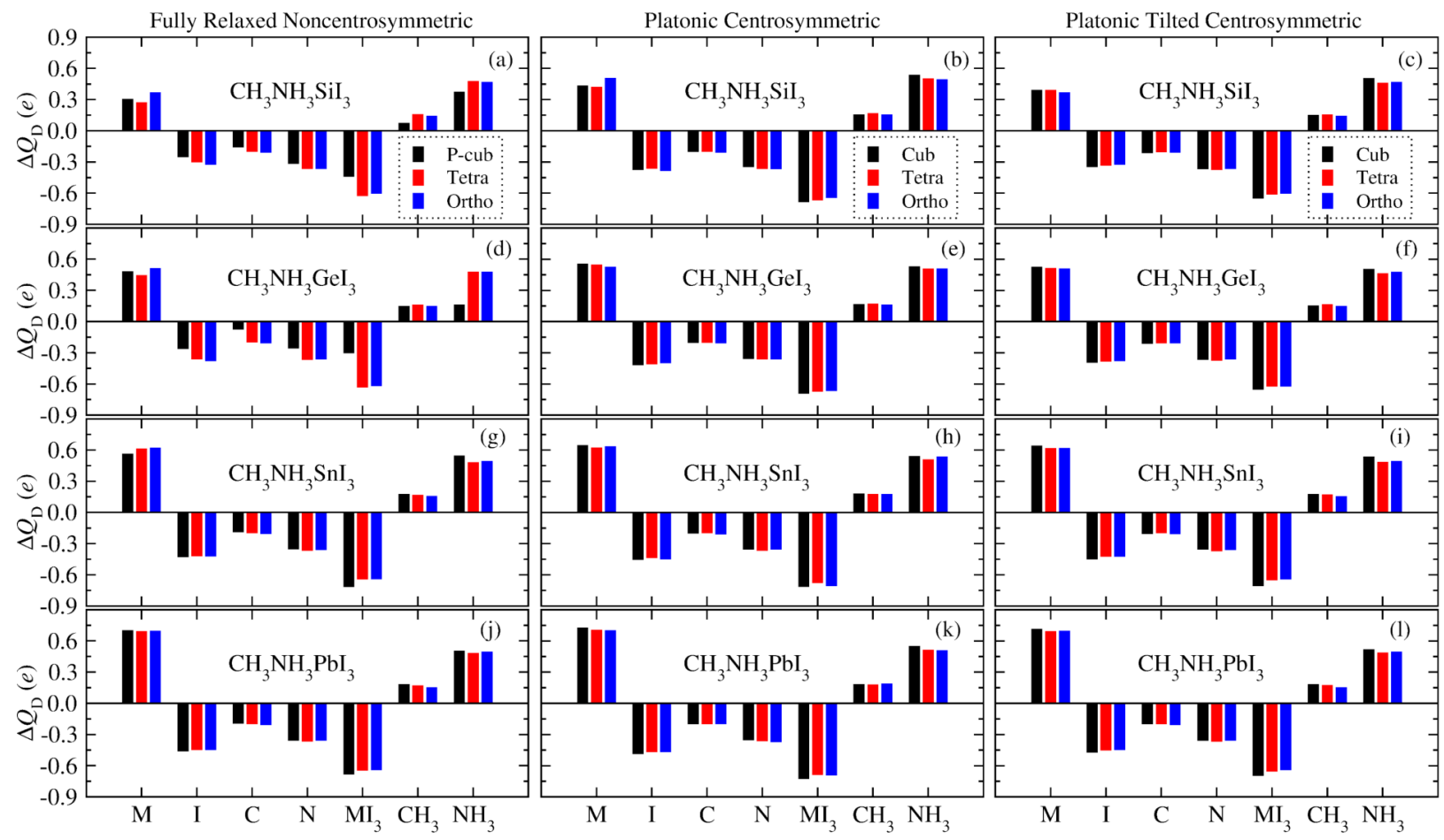

Figure S1 - Partial charges computed for $\mathrm{M}, \mathrm{I}, \mathrm{C}, \mathrm{N}, \mathrm{MI}_{3}, \mathrm{CH}_{3}$, and $\mathrm{NH}_{3}$ via density-derived electrostatic and chemical (DDEC6) method for the MAPbI ${ }_{3}, \mathrm{MASnI}_{3}, \mathrm{MAGeI}_{3}$, and $\mathrm{MASiI}_{3}$ perovskites fully optimized, platonic centrosymmetric with MA relaxed, and platonic-tilted centrosymmetric with MA relaxed configurations. 


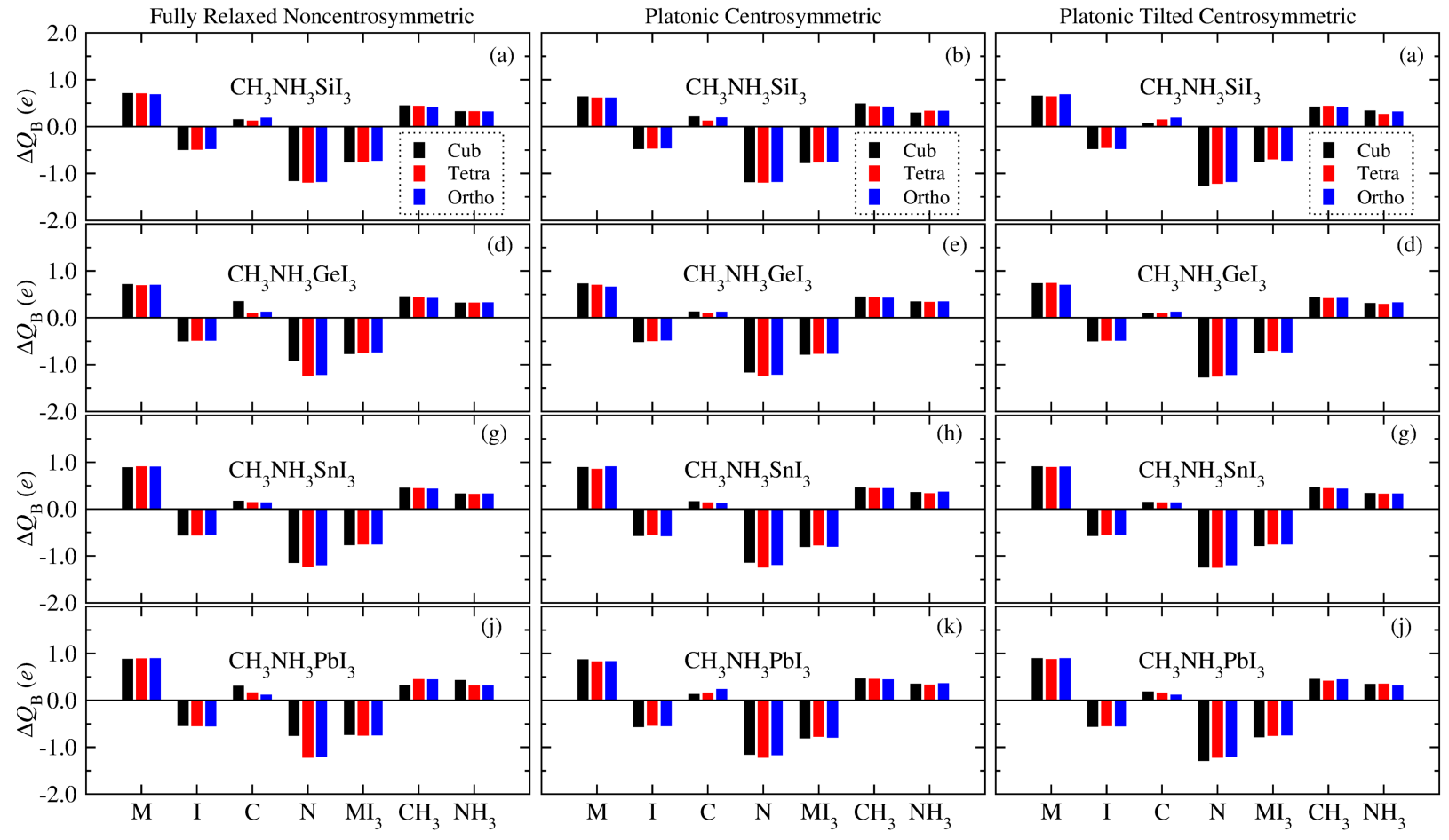

Figure S2 - Partial charges computed for $\mathrm{M}, \mathrm{I}, \mathrm{C}, \mathrm{N}, \mathrm{MI}_{3}, \mathrm{CH}_{3}$, and $\mathrm{NH}_{3}$ via Bader charge method for the $\mathrm{MAPbI}_{3}, \mathrm{MASnI}_{3}, \mathrm{MAGeI}_{3}$, and $\mathrm{MASiI}_{3}$ perovskites fully optimized, platonic centrosymmetric with MA relaxed, and platonic-tilted centrosymmetric with MA relaxed configurations. 

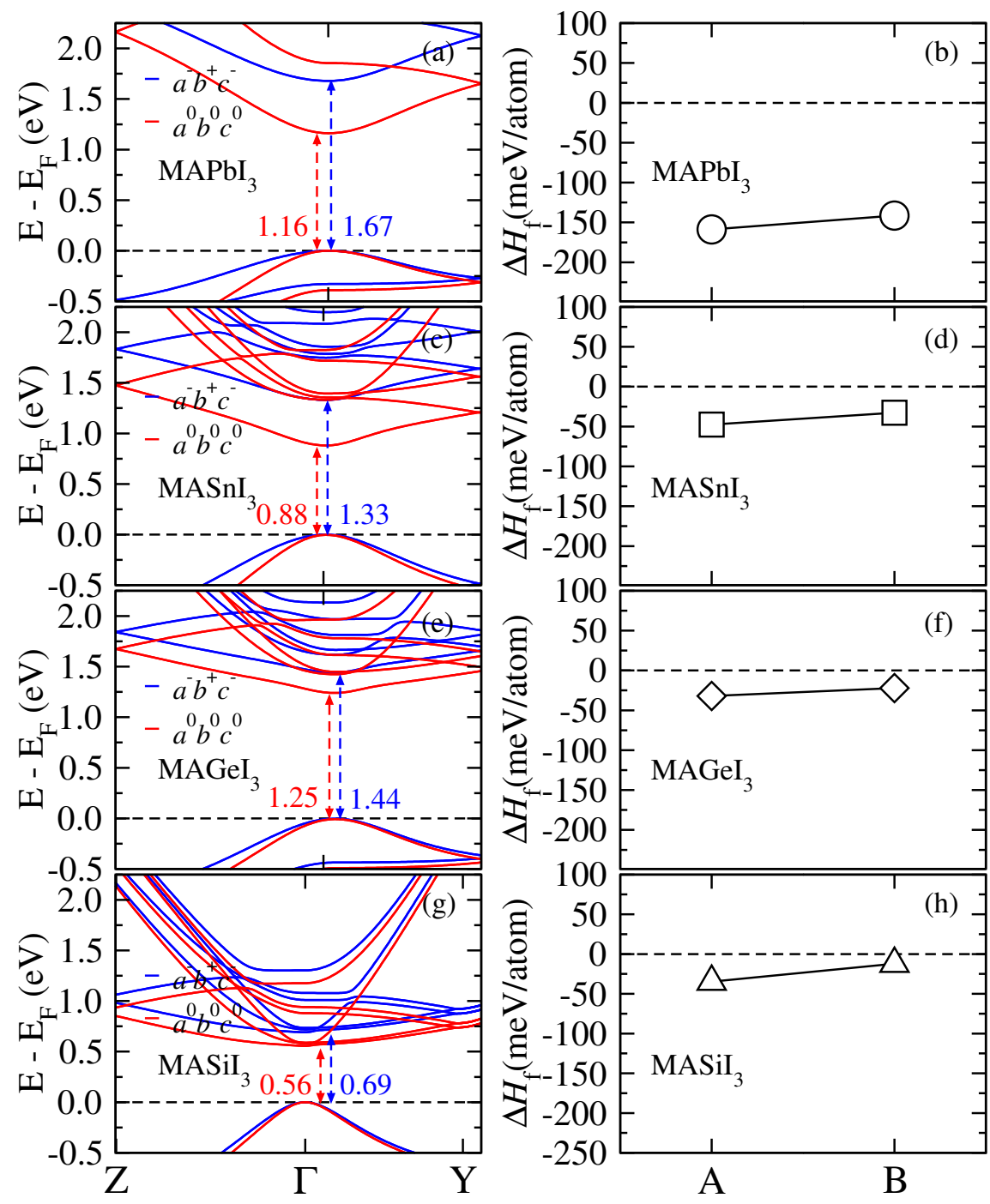

Figure S3 - Band structures and formation enthalpies for $\mathrm{MASiI}_{3}(\mathrm{a}, \mathrm{b}), \mathrm{MAGeI}_{3}(\mathrm{c}, \mathrm{d}), \mathrm{MASnI}_{3}(\mathrm{e}, \mathrm{f})$, and $\operatorname{MAPbI}_{3}(\mathrm{~g}, \mathrm{~h})$ perovskites in orthorhombic $\left(\mathrm{A}: \boldsymbol{a}^{-} b^{+} c^{-}, \mathrm{B}: a^{0} a^{0} a^{0}\right)$ structure. 


\section{References}

(1) Ferreira, L. G.; Marques, M.; Teles, L. K. Approximation to Density Functional Theory for the Calculation of Band Gaps of Semiconductors. Phys. Rev. B 2008, 78, 125116.

(2) Tao, S. X.; Cao, X.; Bobbert, P. A. Accurate and Efficient Band Gap Predictions of Metal Halide Perovskites Using the DFT-1/2 Method: GW Accuracy with DFT Expense. Sci. Rep. 2017, 7, 14386.

(3) Rodrigues Pela, R.; Gulans, A.; Draxl, C. The LDA-1/2 Method Applied to Atoms and Molecules. J. Chem. Theory Comput. 2018, 14, 4678-4686.

(4) Ataide, C. A.; Pelá, R. R.; Marques, M.; Teles, L. K.; Furthmüller, J.; Bechstedt, F. Fast and Accurate Approximate Quasiparticle Band Structure Calculations of $\mathrm{ZnO}, \mathrm{CdO}$, and MgO Polymorphs. Phys. Rev. B 2017, 95, 045126.

(5) Guilhon, I.; Koda, D. S.; Ferreira, L. G.; Marques, M.; Teles, L. K. Approximate Quasiparticle Correction for Calculations of the Energy Gap in Two-Dimensional Materials. Phys. Rev. B 2018, 97, 045426.

(6) Guedes-Sobrinho, D.; Guilhon, I.; Marques, M.; K. Teles, L. Relativistic DFT-1/2 Calculations Combined with a Statistical Approach for Electronic and Optical Properties of Mixed Metal Hybrid Perovskites. J. Phys. Chem. Lett. 2019, 10, 4245-4251.

(7) Ling, C.; Zhao, Z.; Hu, X.; Wang, D.; Li, J.; Zhao, X.; Zhao, Y.; Jin, H. First-Principle Calculation of Electronic and Optical Properties of $\mathrm{VO}_{2}$ by GGA-1/2 Quasiparticle Approximation. J. Appl. Phys. 2020, 128, 235705.

(8) Slater, J. C.; Johnson, K. H. Self-Consistent-Field X $\alpha$ Cluster Method for Polyatomic Molecules and Solids. Phys. Rev. B 1972, 5, 844-853.

(9) Jiang, L.; Wu, T.; Sun, L.; Li, Y.-J.; Li, A.; Lu, R.; Zou, K.; Deng, W.-Q. First-Principles Screening of LeadFree Methylammonium Metal Iodine Perovskites for Photovoltaic Application. J. Phys. Chem. C 2017, 121, 24359-24364.

(10) Hao, F.; Stoumpos, C. C.; Cao, D. H.; Chang, R. P. H.; Kanatzidis, M. G. Lead-Free Solid-State Organic-Inorganic Halide Perovskite Solar Cells. Nat. Photon. 2014, 8, 489-494.

(11) Hao, F.; Stoumpos, C. C.; Chang, R. P. H.; Kanatzidis, M. G. Anomalous Band Gap Behavior in Mixed Sn and Pb Perovskites Enables Broadening of Absorption Spectrum in Solar Cells. J. Am. Chem. Soc. 2014, 136, 8094-8099.

(12) Stoumpos, C. C.; Frazer, L.; Clark, D. J.; Kim, Y. S.; Rhim, S. H.; Freeman, A. J.; Ketterson, J. B.; Jang, J. I.; Kanatzidis, M. G. Hybrid Germanium Iodide Perovskite Semiconductors: Active Lone Pairs, Structural Distortions, Direct and Indirect Energy Gaps, and Strong Nonlinear Optical Properties. J. Am. Chem. Soc. 2015, 137, 68046819.

(13) Yamada, Y.; Nakamura, T.; Endo, M.; Wakamiya, A.; Kanemitsu, Y. Near-Band-Edge Optical Responses of Solution-Processed Organic-Inorganic Hybrid Perovskite $\mathrm{CH}_{3} \mathrm{NH}_{3} \mathrm{PbI}_{3}$ on Mesoporous $\mathrm{TiO}_{2}$ electrodes. Appl. Phys. Express 2014, 7, 032302. 
(14) Leguy, A. M. A.; Azarhoosh, P.; Alonso, M. I.; Campoy-Quiles, M.; Weber, O. J.; Yao, J.; Bryant, D.; Weller, M. T.; Nelson, J.; Walsh, A.; van Schilfgaarde, M.; et al., Experimental and Theoretical Optical Properties of Methylammonium Lead Halide Perovskites. Nanoscale 2016, 8, 6317-6327.

(15) Phuong, L. Q.; Yamada, Y.; Nagai, M.; Maruyama, N.; Wakamiya, A.; Kanemitsu, Y. Free Carriers versus Excitons in $\mathrm{CH}_{3} \mathrm{NH}_{3} \mathrm{PbI}_{3}$ Perovskite Thin Films at Low Temperatures: Charge Transfer from the Orthorhombic Phase to the Tetragonal Phase. J. Phys. Chem. Lett. 2016, 7, 2316-2321.

(16) Fujiwara, H.; Collins, R. W. Spectroscopic Ellipsometry for Photovoltaics: Volume 1: Fundamental Principles and Solar Cell Characterization; Springer, 2018.

(17) Stoumpos, C. C.; Malliakas, C. D.; Kanatzidis, M. G. Semiconducting Tin and Lead Iodide Perovskites with Organic Cations: Phase Transitions, High Mobilities, and Near-Infrared Photoluminescent Properties. Inorg. Chem. 2013, 52, 9019-9038.

(18) Singh, S.; Li, C.; Panzer, F.; Narasimhan, K. L.; Graeser, A.; Gujar, T. P.; Khler, A.; Thelakkat, M.; Huettner, S.; Kabra, D. Effect of Thermal and Structural Disorder on the Electronic Structure of Hybrid Perovskite Semiconductor $\mathrm{CH}_{3} \mathrm{NH}_{3} \mathrm{PbI}_{3}$. J. Phys. Chem. Lett. 2016, 7, 3014-3021.

(19) Kittel, C. Introduction to Solid State Physics, 8th Ed.; John Wiley \& Sons, Inc., New York, 2004.

(20) Manz, T. A.; Limas, N. G. Introducing DDEC6 Atomic Population Analysis: Part 1. Charge Partitioning Theory and Methodology. RSC Adv. 2016, 6, 47771-47801.

(21) Bader, R. F. W. Atoms in Molecules: A Quantum Theory; International Series of Monographs on Chemistry; Clarendon Press, 1994.

(22) Pauling, L. The Nature of the Chemical Bond; Cornell University Press: Ithaca, 1960. 